\title{
The role of serum bile acid profile in differentiation between nonalcoholic fatty liver disease and chronic viral hepatitis
}

\author{
Azza Elsheashaey ${ }^{1,2}$, Manar Obada $^{1}$, Eman Abdelsameea ${ }^{3^{*}} \mathbb{D}$, Mohamed F. F. Bayomy ${ }^{2}$ and Hala El-Said ${ }^{1}$
}

\begin{abstract}
Background: Bile acids are essential organic molecules synthesized from cholesterol in the liver. They have been utilized as indicators of hepatobiliary impairment because synthesis of BAs and their metabolism are influenced by liver diseases. We aimed to investigate the role of serum bile acid level and composition in differentiation between nonalcoholic fatty liver disease (NAFLD) and chronic viral hepatitis. An ultra-performance liquid chromatography coupled with mass spectrometry assay was used to measure the serum level of 14 bile acids in chronic viral hepatitis and NAFLD patients beside normal healthy control subjects.

Results: The mean serum levels of 11 out of the 14 bile acids (two primary, six conjugated, and three secondary) were significantly higher in viral hepatitis compared to control. Only 4 bile acids [2 primary, one glycine conjugated (GCDCA), and one secondary (LCA)] had statistically significant increase in their mean serum bile acid level in NAFL D compared to control. Comparing viral hepatitis group against NAFLD group revealed that the mean serum levels of five conjugated and one secondary bile acid (DCA) were significantly higher in viral hepatitis group. Receiver operating characteristic (ROC) curve analysis revealed that LCA had the best diagnostic performance for viral hepatitis followed by TCA and GCDCA. ROC curve for the combined three parameters had better sensitivity and specificity (70.55\% and $94.87 \%$ respectively).
\end{abstract}

Conclusion: BA compositions including primary, secondary, and conjugated ones could differentiate between chronic viral hepatitis and NAFLD patients, and they might be potential distinguishing biomarkers for this purpose.

Keywords: Bile acids (BAs), Nonalcoholic fatty liver disease (NAFLD), Chronic viral hepatitis, HCV, HBV

\section{Background}

Bile acids (BAs) are synthesized in the liver from cholesterol, and they are essential component of bile. BAs are synthesized in the liver as primary bile acids chenodeoxycholic acid (CDCA) and cholic acid (CA). They are completely conjugated with taurine or glycine to form tauroconjugates and glycoconjugates respectively before being secreted into the biliary tree. The conjugated primary bile acids are known as taurocholic acid (TCA), glycocholic acid (GCA), taurochenodeoxycholic acid

\footnotetext{
* Correspondence: dreman555@yahoo.co.uk

${ }^{3}$ Department of Hepatology and Gastroenterology, National Liver Institute,

Menoufia University, Shebeen El-Kom 32511, Egypt

Full list of author information is available at the end of the article
}

(TCDCA), and glycochenodeoxycholic acid (GDCA). All these conjugated bile acids are named bile salt [1]. They were modified by intestinal bacteria after their secretion into the small intestine into secondary bile acids lithocholic acid (LCA), deoxycholic acid (DCA), and ursodeoxycholic acid (UDCA) [2].

Hepatitis C virus (HCV) and hepatitis B virus (HBV) infections are the most common leading causes of chronic hepatitis developing into liver cirrhosis (LC) with or without hepatocellular carcinoma (HCC) [3].

Nonalcoholic fatty liver disease (NAFLD) is a spectrum of several metabolic disorders which start with simple steatosis that has excessive triglyceride accumulation in hepatocytes which progresses to nonalcoholic

\section{Springer Open}

(- The Author(s). 2020 Open Access This article is licensed under a Creative Commons Attribution 4.0 International License, which permits use, sharing, adaptation, distribution and reproduction in any medium or format, as long as you give appropriate credit to the original author(s) and the source, provide a link to the Creative Commons licence, and indicate if changes were made. The images or other third party material in this article are included in the article's Creative Commons licence, unless indicated otherwise in a credit line to the material. If material is not included in the article's Creative Commons licence and your intended use is not permitted by statutory regulation or exceeds the permitted use, you will need to obtain permission directly from the copyright holder. To view a copy of this licence, visit http://creativecommons.org/licenses/by/4.0/. 
steatohepatitis (NASH) with inflammation, fibrosis, and cirrhosis, and development of liver cell failure and HCC. The mechanism of progression of simple steatosis to steatohepatitis is not clear entirely although several pathways have been suggested. Disruption of bile acid homeostasis is one of the common links among these several pathways [4].

Bile acid homeostasis under physiological condition is maintained by multiple negative feedback loops for synthesis of bile acid [5] and very strictly regulated bile acid enterohepatic circulation [6].

Because synthesis of BAs and their metabolism are influenced by liver diseases, BAs and also their composition have been used as prognostic and diagnostic markers. However, it is unclear how causes of liver disease affect the composition of BA [7]. In this regard, the present study aimed to investigate the serum BA compositions, including the levels of primary, secondary, and conjugated BAs, using ultra-performance liquid chromatography tandem mass spectrometer (UPLC-MS/MS) in a number of patients with chronic viral hepatitis and NAFLD in addition to healthy control, and also to study how bile acid composition can differentiate between chronic viral hepatitis and NAFLD patients and possibility of individual bile acids (IBA) and their profiles to be potential biomarkers for this purpose.

\section{Methods}

\section{Chemicals and reagents}

Methanol, acetonitrile, and formic acid were HPLC grade and purchased from Fisher Scientific (Loughborough, UK). Bile acid standards: cholic acids (CA), chenodeoxycholic acid (CDCA), deoxycholic acid (DCA), lithocholic acid (LCA), ursodeoxycholic acid (UDCA), glycocholic acid (GCA), glycochenodeoxycholic acid (GCDCA), glycodeoxycholic acid (GDCA), glycoursodeoxycholic acid (GUDCA), taurocholic acid (TCA), taurochenodeoxycholic acid (TCDCA), taurodeoxycholic acid (TDCA), tauroursodeoxycholic acid (TUDCA), and taurolithocholic acid (TLCA), were also purchased from Sigma Chemical Sigma-Aldrich (Merck KGaA, Darmstadt, Germany). HPLC grade water was obtained from Millipore pure water purification system (Diamond TII, USA).

\section{Patients}

The study was conducted in the period from October 2017 to August 2019. The study enrolled 2 groups of patients: chronic viral hepatitis (group 2) included 146 patients and their mean ages were $46.0(39-51)$ years old, and NAFLD (group 3) included 39 patients and their mean ages were $47(41.5-52)$ years old, beside normal healthy control subjects included 51 individuals (group $1)$ and their mean ages were $47.0(38-52.5)$ years old.
Patients in the chronic viral hepatitis group had chronic HBV or chronic HCV infection. Their diagnosis was based on positive hepatitis B surface antigen (HBsAg) and detectable HBV DNA and positive $\mathrm{HCV}$ antibody and detectable HCV RNA for more than 6 months respectively. In addition to established clinical, laboratory, and imaging findings of liver cirrhosis with no evidence of any hepatic focal lesion at the time of enrolment, NAFLD diagnosis was based on imaging analysis such as abdominal ultrasound and liver biopsy. All liver biopsy specimens at least $25 \mathrm{~mm}$ in length were obtained by percutaneous route. Liver sections were stained routinely with hematoxylin and eosin, Masson trichrome, silver reticulin, and occasionally with diastase-resistant periodic acid-Schiff and Perls' Prussian blue. Liver biopsies had been read by a single pathologist who estimated semi-quantitatively the histopathological changes according to Brunt classification [8]. Fifty one normal healthy subjects were included matching the age and the gender of the other groups, with no clinical, laboratory, or radiological evidence of any type of liver diseases.

\section{Ethical considerations}

The study was conducted according to ethical standards for human experimentation (Helsinki Declaration). The ethics committee of the National Liver Institute approved the protocol, and written consents were filled and signed by all participants.

\section{Sample collection}

A sample $(5 \mathrm{ml})$ of fasting venous blood was obtained in the early morning from each patient and control subject and divided into two tubes. Two millimeters was collected into an EDTA-containing tube for CBC assessment. Three millimeters was collected in plain vacutainer tube, after coagulation, and centrifugation of the sera was separated into aliquots for measurement of liver function tests and stored at $-80^{\circ} \mathrm{C}$ until UPLCanalysis.

\section{Laboratory investigations}

Serum biochemical assay was performed with an automatic biochemistry analyzer (Daytona plus, Randox laboratories limited, UK) for analysis of blood chemistry as liver function tests including albumin, aspartate aminotransferase (AST), alanine aminotransferase (ALT), bilirubin total, alkaline phosphatase (ALP), and gamma glutamyl transpeptidase (GGT). Alfafetoprotein (AFP) was performed by using (ARCHITECTi1000SR immunoassay analyzer, Abbott, Abbott Park, IL, USA). Hematological parameters and blood films were performed and measured using automatic analyzer (Sysmex KX-21, SysmexInc., Japan). The biochemical determinations were performed on the same day as blood was taken. 


\section{Serum sample preparation and bile acid detection} Sample preparation

The sample preparation method was based on a published method of [7] with modification. First, we added $100 \mu \mathrm{L}$ of serum sample to $400 \mu \mathrm{L}$ of ice cold methanol to precipitate proteins, vortex the mixture, then centrifugation of the mixture at 13,500 rpm for 15 min occurred; then, we separated the supernatant in a clean eppendorf bottle and centrifuged again at 13,500 rpm for $15 \mathrm{~min}$; finally, $50 \mu \mathrm{L}$ of the supernatant with $100 \mu \mathrm{L}$ water/formic acid (1000: 1, v/v) solution was injected into the LC/MS/ MS system. All chromatographic separations were performed with an ACQUITY HSS C18 column $(1.7 \mu \mathrm{m}$, $100 \mathrm{~mm} \times 2.1 \mathrm{~mm}$ internal dimensions) (Waters, Milford, MA). The injected sample volume was $10 \mu \mathrm{L}$, and the column temperature was maintained at $50{ }^{\circ} \mathrm{C}$. Individual bile acids were eluted with a gradient at a flow rate of $0.5 \mathrm{ml} / \mathrm{min}$.

Mobile phase A was (1/1000) formic acid/water, and mobile phase $\mathrm{B}$ was acetonitrile. The samples were eluted with $80 \%$ mobile phase A and $20 \%$ mobile phase $B$ for an initial 2 min after injection, then with a linear gradient of mobile phase B of 20 to $30 \%$ over $5 \mathrm{~min}$, followed by mobile phase B at $80 \%$ over $8 \mathrm{~min}$, which was held for $0.50 \mathrm{~min}$. Before the injection of the next sample, the column was equilibrated with $80 \%$ mobile phase A for $2 \mathrm{~min}$. The mass spectrometer had an electrospray source operated in the negative ion mode using the multiple reaction monitoring (MRM) mode. UPLCMS raw data obtained with MRM mode were analyzed using MassLynx applications manager version 4.1 (Waters Corp., Milford, MA) to obtain the calibration equations and the quantitative concentration of each bile acid in the samples. The method was validated ranging from 0.0010 to $20 \mathrm{umol} / \mathrm{L}$.

\section{Statistical analysis}

Data were analyzed using the IBM SPSS software package version 20.0. (Armonk, NY: IBM Corp). Qualitative data were described using number and percent. The Kolmogorov-Smirnov test was used to verify the normality of distribution. Quantitative data were described using range (minimum and maximum), mean, standard deviation, and median. Significance of the obtained results was judged at the $5 \%$ level. The used tests were Chi-square test, for categorical variables, to compare between different groups; Monte Carlo correction for chisquare when more than $20 \%$ of the cells have expected count less than 5; $F$ test (ANOVA), for normally distributed quantitative variables, to compare between more than two groups; post hoc test (Tukey) for pairwise comparisons; Kruskal-Wallis test for abnormally distributed quantitative variables to compare between more than two studied groups; and post hoc (Dunn's multiple comparison test) for pairwise comparisons.

\section{Results \\ Clinical patient characteristics}

A total of 185 patients were enrolled in this study. The chronic viral hepatitis (group 2) included 146 patients [60 (41.1\%) were male]; there were $45(30.8 \%)$ who had chronic HBV and 101 (69.2\%) had chronic HCV (39\% were cirrhotic), and in 39 NAFLD patients (group 3) [30 (76.9\%) were female], $20.5 \%$ of them were cirrhotic.

\section{Biochemical data}

The results of biochemical data in all studied groups are shown in Table 1. Significantly higher serum levels of AST, ALT, GGT, and ALP and significantly lower serum levels of albumin were found in viral hepatitis and NAFL D groups compared to control, and significantly higher levels of serum total bilirubin and AFP was found in viral hepatitis group compared to NAFLD group ( $p=$ $0.042,<0.001$ respectively).

\section{Bile acid profiles in viral hepatitis and NAFLD groups compared to healthy control}

A total of 14 bile acids were determined and compared across the three groups. These include two primary (CA, CDCA), 5 taurine-conjugated (TCA, TCDCA, TUDCA TDCA, TLCA), 4 glycineconjugated (GCA, GCDCA, GUDCA GDCA), and 3 secondary (UDCA, DCA, LCA) bile acids. The comparisons of these bile acids among the different groups are summarized in Table 2 . The mean serum levels of 11 out of the 14 bile acids, 2 primary (CA, CDCA), 3 taurine-conjugated (TCA, TCDCA, TLCA), 3 glycine-conjugated (GCA, GCDCA, GDCA), and 3 secondary (UDCA, DCA and LCA), were significantly higher in viral hepatitis (group 2) compared to control group (group 1). Three bile acids TUDCA, TDCA, and GUDCA showed no significant difference $(p>0.05)$ among the studied groups.

Comparing NAFLD (group 3) against the control revealed that only 4 bile acids, 2 primary (CA, CDCA) $[p=0.007,0.002$ respectively], 1 glycine conjugated (GCDCA) $[p=0.011]$, and 1 secondary (LCA) $[p<$ $0.001]$, had statistically significant increase in their mean serum bile acid level compared to the control.

\section{Bile acid profiles in viral hepatitis compared to NAFLD group}

Comparing viral hepatitis group against NAFLD group, the mean serum levels of taurine-conjugated bile acids (TCA and TCDCA) [ $p<0.001,0.016$ respectively], glycine conjugated (GCA, GCDCA, and GDCA) $[p=0.003$, $0.002,0.006$ respectively], and DCA (secondary bile acid) 
Table 1 Comparison between the studied groups regarding biochemical data

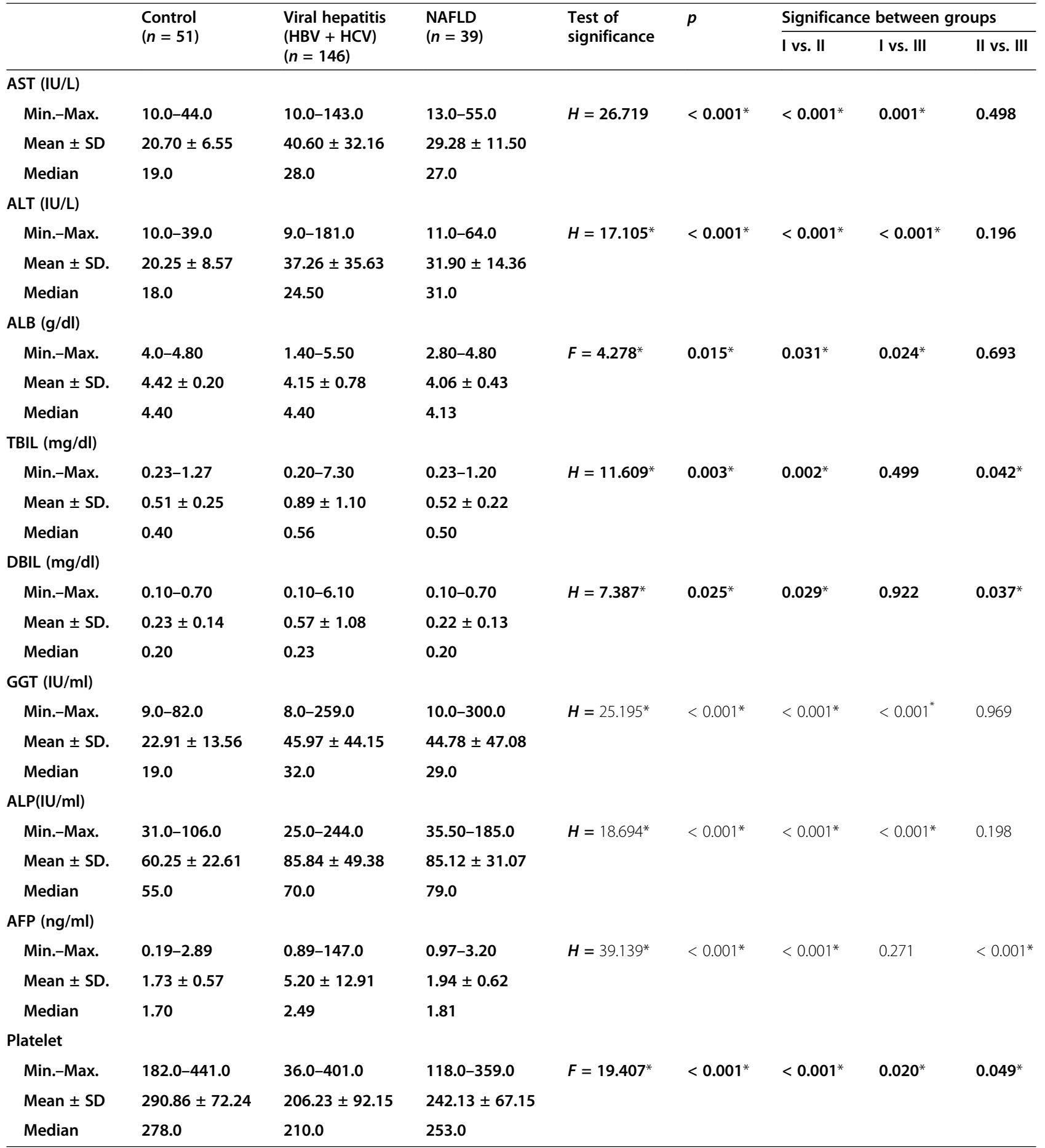

$M$ mean, SD standard deviation, Min. minimum, Max. maximum, $A S T$ aspartate aminotransferase, $A L T$ alanine aminotransferase, $A L B$ albumin, TBIL total bilirubin, $D B I L$ direct bilirubin, GGT gamma glutamyl transferase, ALP alkaline phosphatase, AFP alpha fetoprotein

$H$ : $H$ for Kruskal-Wallis test, pairwise comparison between 2 groups was done using post hoc test (Dunn's for multiple comparisons test), $F: F$ for ANOVA test, pairwise comparison between 2 groups was done using post hoc test (Tukey). $p: p$ value for comparing between the studied groups

*Statistically significant at $p<0.05$

$[p=0.039]$ were significantly higher in the viral hepatitis group. Meanwhile, there was no significant difference between the two groups regarding the mean serum levels of other bile acids $[p>0.05]$ (Table 2).
Serum bile acids to discriminate between viral hepatitis and NAFLD

To assess the ability of serum bile acids to distinguish viral hepatitis group (group 2) from NAFLD group (group 3), a 
Table 2 Comparison of 14 bile acid composition among the three studied groups

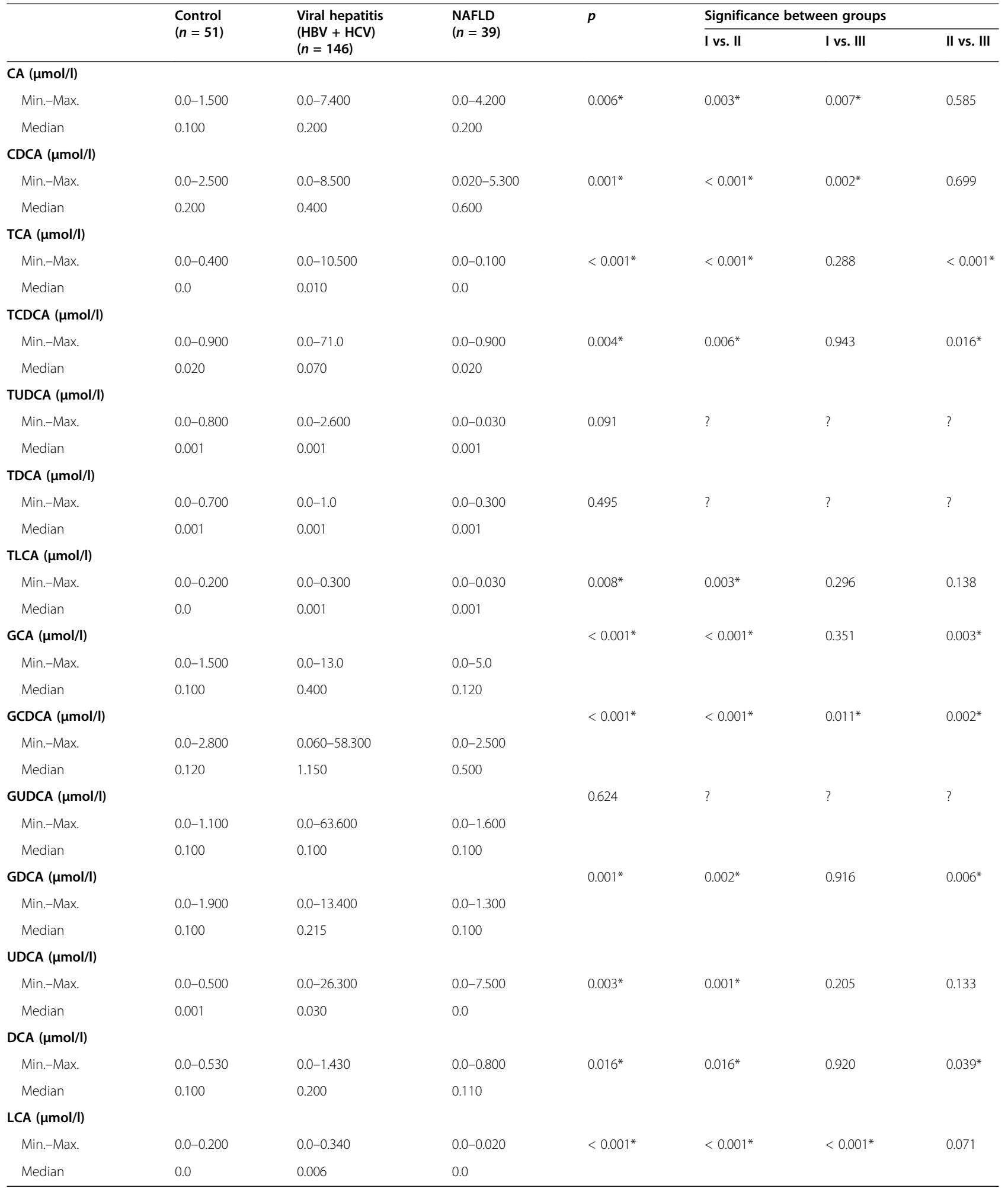

Min. minimum, Max. maximum, CA cholic acid, CDCA chenodeoxycholic acid, TCA taurocholic acid, TCDCA taurochenodeoxycholic acid, TUDCA

tauroursoodeoxycholic acid, TDCA taurodeoxycholic acid, TLCA taurolithocholic acid, GCA glycholic acid, GCDCA glycochenodeoxycholic acid, GUDCA glycoursodeoxycholic acid, GDCA glycodeoxycholic acid, UDCA ursodeoxycholic acid, DCA deoxycholic acid, LCA lithocholic acid, Vs. versus

$H: H$ for Kruskal-Wallis test, pairwise comparison between each 2 groups was done using post hoc test (Dunn's for multiple comparisons test) $p: p$ value for comparing between the studied groups

*Statistically significant at $p<0.05$ 


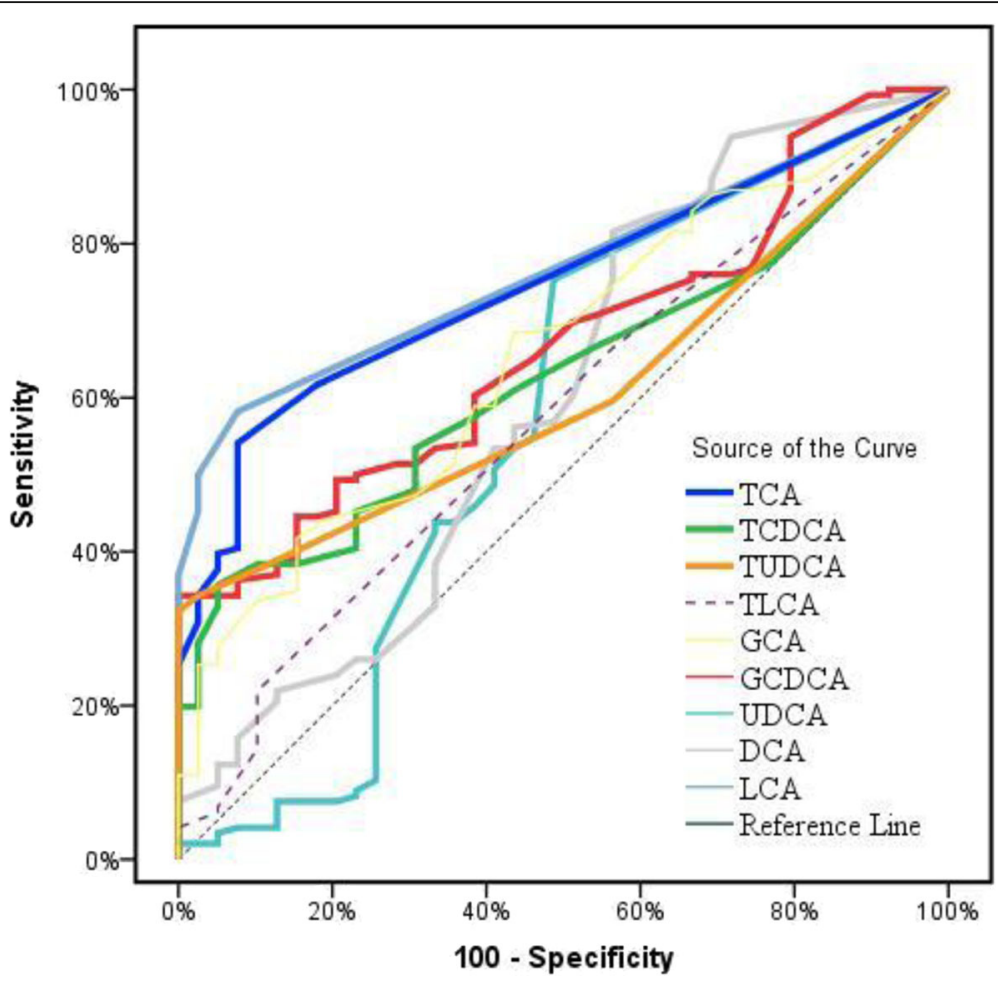

Fig. 1 ROC curve analysis of different bile acids to distinguish viral hepatitis group from NAFLD group

receiver operating characteristic (ROC) curve analysis of the TCA, TCDCA, TUDCA, TLCA, GCA, GCDCA, UDCA, DCA, and LCA bile acids was performed as shown in Fig. 1 and Table 3. The ROC curves revealed that LCA had the best diagnostic performance with an area under the receiver operating characteristic curve (AUROC) of 0.769 [95\% confidence interval $(\mathrm{CI}), 0.70-0.83 ; p<0.001$ ] and sensitivity and specificity were $58.22 .5 \%$ and $92.31 \%$ respectively followed by TCA with AUROC of 0.749 (95\%
CI, 0.68-0.81; $p<0.001)$ and GCDCA with AUROC 0.667 (95\% CI, 0.59-0.73; $p=0.001$ ) (Table 3).

To assess the ability of combined LCA, TCA, and GCDCA to distinguish viral hepatitis from NAFLD, a ROC curve for the combined three parameters was performed revealing that these parameters together were good predictors for chronic viral hepatitis with AUROC 0.847 as shown in Table 4 and Fig. 2, and sensitivity and specificity were $70.55 \%$ and $94.87 \%$ respectively.

Table 3 Sensitivity, specificity, and diagnostic accuracy of serum bile acids levels to distinguish viral hepatitis from NAFLD

\begin{tabular}{|c|c|c|c|c|c|c|c|c|}
\hline \multicolumn{9}{|c|}{ Viral hepatitis (group 2) versus NAFLD (group 3) } \\
\hline & AUC & $p$ & 95\% C.I & Cutoff & Sensitivity & Specificity & PPV & NPV \\
\hline TCA & $0.749^{*}$ & $<0.001^{*}$ & $0.68-0.81$ & $>0.001$ & 54.11 & 92.31 & 96.3 & 35.0 \\
\hline TCDCA & $0.627^{*}$ & $0.003^{*}$ & $0.55-0.70$ & $>0.02$ & 60.96 & 56.41 & 84.0 & 27.8 \\
\hline TUDCA & $0.608^{*}$ & $0.006^{*}$ & $0.55-0.70$ & $>0$ & 59.59 & 43.59 & 79.8 & 22.4 \\
\hline TLCA & 0.575 & 0.108 & $0.50-0.65$ & $>0$ & 66.44 & 43.59 & 81.5 & 25.8 \\
\hline GCA & 0.657 & $0.006^{*}$ & $0.58-0.73$ & $>0.35$ & 51.37 & 64.10 & 84.3 & 26.0 \\
\hline GCDCA & 0.667 & $0.001^{*}$ & $0.59-0.73$ & $>1.1$ & 50.0 & 76.92 & 89.0 & 29.1 \\
\hline UDCA & 0.570 & 0.182 & $0.45-0.69$ & $>0$ & 75.34 & 51.28 & 85.3 & 35.7 \\
\hline DCA & 0.602 & $0.049^{*}$ & $0.49-0.71$ & $>0.04$ & 81.51 & 43.59 & 84.4 & 38.6 \\
\hline LCA & 0.769 & $<0.001^{*}$ & $0.70-0.83$ & $>0$ & 58.22 & 92.31 & 96.6 & 37.1 \\
\hline
\end{tabular}

AUC area under a curve, $p$ value probability value, $C I$ confidence intervals, NPV negative predictive value, $P P V$ positive predictive value, $C D C A$ chenodeoxycholic acid, TCA taurocholic acid, TCDCA taurochenodeoxycholic acid, TUDCA taurodeoxycholic acid, TLCA taurolithocholic acid, GCA glycholic acid, GCDCA glycochenodeoxycholic acid, UDCA ursodeoxycholic acid, DCA deoxycholic acid, LCA lithocholic acid *Statistically significant at $p<0.05$ 
Table 4 Sensitivity, specificity, and diagnostic accuracy of combined TCA, LCA, and GCDCA to distinguish viral hepatitis group from NAFLD group

\begin{tabular}{lllll}
\hline Viral hepatitis (group 2) versus NAFLD (group 3) & & \\
\hline & Sensitivity & Specificity & PPV & NPV \\
\hline TCA + LCA + GCDCA & 70.55 & 94.87 & 98.1 & 46.2
\end{tabular}

AUC area under a curve, $p$ value probability value, $C$ c confidence intervals, NPV negative predictive value, PPV positive predictive value, TCA taurocholic acid, $L C A$ lithocholic acid, GCDCA glycochenodeoxycholic acid

*Statistically significant at $p<0.05$

In comparing viral hepatitis group versus NAFLD group regarding the cutoff values of their serum bile acid levels, we found that serum levels of TCA, GCDCA, UDCA, DCA, and LCA were significantly higher in viral hepatitis group $(p<0.001, p=0.003, p=0.001, p=0.001$, and $p<0.001$ respectively) as shown in Table 5 .

\section{Discussion}

$\mathrm{HCV}$ is a major health problem worldwide with 70-100 million people have chronic $\mathrm{HCV}$ infection and subsequently leads to cirrhosis and hepatocellular carcinoma [9]. Also, hepatitis B virus makes major health problem. Chronic HBV infection may develop cirrhosis and subsequently liver decompensation and hepatocellular carcinoma which is a major drastic complication [10]. Recently, nonalcoholic fatty liver disease is considered the most common cause of liver disease with prevalence of $25 \%$ worldwide. Patients with NAFLD with proved nonalcoholic steatohepatitis and advanced fibrosis are at marked increase in the risk of adverse outcomes, including liver-specific morbidity and mortality and overall mortality [11]. Liver biopsy (LB) is the gold standard for NASH diagnosis and assessment of the fibrosis stage in patients with NAFLD in spite of its many limitations including cost, sampling error, morbidity, and death in very rare cases [12]. As liver diseases affect BA metabolism, the composition of BAs was studied and used as diagnostic markers. However, it is not clear how different etiologies of chronic liver diseases may affect the composition of BA [7]. In this study, we investigated the serum BA composition using LC-MS/MS in a number of chronic viral hepatitis and NAFLD patients in addition to healthy controls to show if bile acids can be used as diagnostic markers for distinguishing between chronic viral hepatitis and NAFL D. In our study, the mean serum levels of 2 primary (CA, CDCA), 3 taurine conjugated (TCA, TCDCA, TLCA), 3 glycine conjugated (GCA, GCDCA, GDCA) and 3 secondary (UDCA, DCA and LCA) were significantly higher in patients with chronic viral hepatitis compared to the control group. These results were in agreement with Luo et al. where they found that concentrations of individual bile acids (IBA) in patients with liver impairments as in hepatitis $B$ and $C$ were significantly higher when

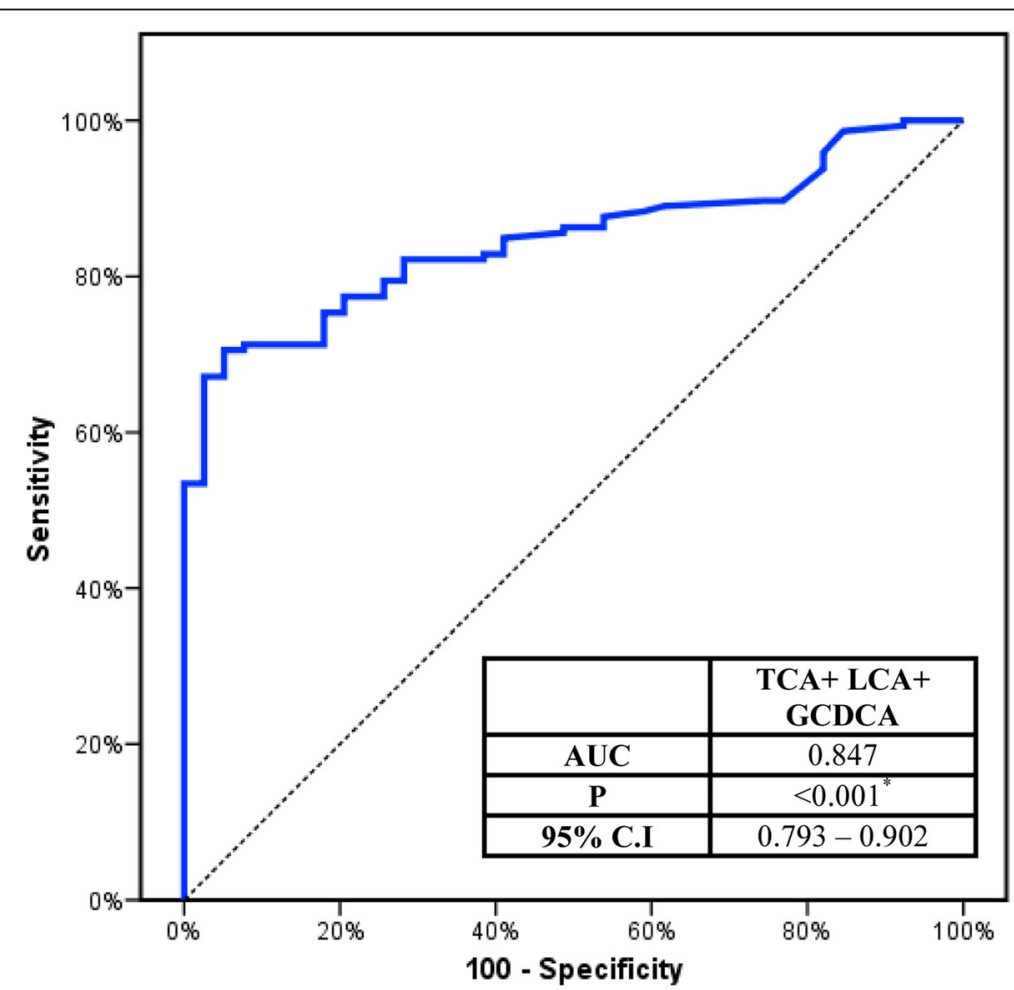

Fig. 2 ROC curve analysis of combined TCA, LCA, and GCDCA to distinguish viral hepatitis group from NAFLD group 
Table 5 Comparison between viral hepatitis and NAFLD groups according to the cutoff values of their bile acids

\begin{tabular}{|c|c|c|c|c|c|c|}
\hline & \multicolumn{2}{|c|}{ Viral hepatitis (group II) $(n=146)$} & \multicolumn{2}{|c|}{ NAFLD (group III) $(n=39)$} & \multirow[t]{2}{*}{$x^{2}$} & \multirow[t]{2}{*}{$p$} \\
\hline & No. & $\%$ & No. & $\%$ & & \\
\hline \multicolumn{7}{|l|}{ TCA } \\
\hline$\leq 0.001$ & 67 & 45.9 & 36 & 92.3 & $26.872^{*}$ & $<0.001^{*}$ \\
\hline$>0.001$ & 79 & 54.1 & 3 & 7.7 & & \\
\hline \multicolumn{7}{|l|}{ TCDCA } \\
\hline$\leq 0.02$ & 57 & 39.0 & 22 & 56.4 & 3.795 & 0.051 \\
\hline$>0.02$ & 89 & 61.0 & 17 & 43.6 & & \\
\hline \multicolumn{7}{|l|}{ TUDCA } \\
\hline$\leq 0$ & 59 & 40.4 & 17 & 43.6 & 0.128 & 0.720 \\
\hline$>0$ & 87 & 59.6 & 22 & 56.4 & & \\
\hline \multicolumn{7}{|l|}{ TLCA } \\
\hline$\leq 0$ & 49 & 33.6 & 17 & 43.6 & 1.349 & 0.245 \\
\hline$>0$ & 97 & 66.4 & 22 & 56.4 & & \\
\hline \multicolumn{7}{|l|}{ GCA } \\
\hline$\leq 0.35$ & 71 & 48.6 & 25 & 64.1 & 2.952 & 0.086 \\
\hline$>0.35$ & 57 & 51.4 & 14 & 35.9 & & \\
\hline \multicolumn{7}{|l|}{ GCDCA } \\
\hline$\leq 1.1$ & 73 & 50.0 & 30 & 76.9 & $9.040^{*}$ & $0.003^{*}$ \\
\hline$>1.1$ & 73 & 50.0 & 9 & 23.1 & & \\
\hline \multicolumn{7}{|l|}{ UDCA } \\
\hline$\leq 0$ & 36 & 24.7 & 20 & 51.3 & $10.337^{*}$ & $0.001^{*}$ \\
\hline$>0$ & 110 & 75.3 & 19 & 48.7 & & \\
\hline \multicolumn{7}{|l|}{ DCA } \\
\hline$\leq 0.04$ & 27 & 18.5 & 17 & 43.6 & $10.694^{*}$ & $0.001^{*}$ \\
\hline$>0.04$ & 119 & 81.5 & 22 & 56.4 & & \\
\hline \multicolumn{7}{|l|}{ LCA } \\
\hline$\leq 0$ & 61 & 41.8 & 36 & 92.3 & $31.505^{*}$ & $<0.001^{*}$ \\
\hline$>0$ & 85 & 58.2 & 3 & 7.7 & & \\
\hline
\end{tabular}

$x^{2}$ Chi-square test, $p$ value for comparing between the studied groups

CDCA Chenodeoxycholic acid, TCA taurocholic acid, TCDCA taurochenodeoxycholic acid, TUDCA taurodeoxycholic acid, TLCA taurolithocholic acid, GCA glycholic acid, GCDCA, UDCA ursodeoxycholic acid, DCA deoxycholic acid, LCA lithocholic acid

*Statistically significant at $p \leq 0.05$

compared to control [13]. Also, Wang et al. and Yin et al. reported that the serum levels of TCA, TCDCA, GCA, and GCDCA, the conjugated BAs, were significantly increased in cirrhotic patients and conjugated bile acids could be indicators for liver dysfunction in patients with chronic hepatitis [14, 15]. Makino et al. also reported that the concentration of serum BAs are increased in chronic liver diseases especially cirrhotic patients due to the impairment of bile production and secretion [16].

In contrast to our results, Luo et al. found that there was no significant difference between patients with liver impairments as in hepatitis $\mathrm{B}$ and $\mathrm{C}$ and healthy controls as regards DCA. This may be explained by the fact that patients included in their study had liver impairment of non-viral etiology [13].
From previous results, it is clear that the serum concentrations of bile acids were significantly higher in viral hepatitis compared to healthy control and where the serum bile acids concentration reflects how bile acids reabsorbed from intestine could succeed in escape of first extraction through the liver. The efficiency of extraction may be reduced in liver disease as they either decreased functional hepatocyte mass or shunting of blood past hepatocyte and consequently, systemic bile acid levels increased and approach those present normally in portal circulation [7].

We found that only 4 bile acids, 2 primary (CA, CDCA), 1 glycine conjugated (GCDCA), and 1 secondary (DCA), had statistically significant increase in the mean serum levels in NAFLD group compared to 
control. In agreement with our study, Sugita et al. reported that serum bile acids were dysregulated in patients with NAFLD, and primary bile acids (CA and CDCA) increased 3.8-fold in 13 patients with NAFLD compared with 46 healthy control subjects [7].

Similarly, Wang et al. and Minnullina et al. reported that patients with nonalcoholic fatty liver disease had higher bile acid level compared to healthy controls $[17,18]$.

Also, Kalhan et al. reported that metabolomic analysis has revealed significantly increased serum levels of GCDCA in patients with nonalcoholic steatohepatitis compared with healthy controls. Meanwhile they found that TCA and GCA was significantly higher in their patients compared to control, but in our study, there was insignificant difference between the two groups regarding taurocholate (TCA) and glycocholate (GCA). This may be explained that most patients included in our NAFLD group were non-cirrhotic [19].

It remains speculative for explaining the main mechanism responsible for the higher bile acid concentration in patients with hepatic steatosis and $\mathrm{NASH}$. It could be consequence of either increased pool of bile acid due to a higher bile acid synthesis rate, result from increased microsomal and peroxisomal metabolism, might be caused by hepatocellular injury, or probably be an adaptive response to the triglyceride accumulation in the liver. A higher bile acid concentration has been previously found in patients with hyperlipidemia. It is reasonable that triglyceride accumulation in the liver or increased oxidation of fatty acid compromises liver function, resulting in its inefficient bile acid uptake from the circulation. The higher bile acid levels could also be due to the higher insulin resistance in patients with NAFLD. The interaction between hepatic insulin receptors, insulin, and bile acids is complex [19].

In our study, the mean serum levels of taurineconjugated bile acids (TCA and TCDCA), glycine conjugated (GCA, GCDCA, and GDCA) and DCA were significantly higher in viral hepatitis group compared to NAFLD group. Also, LCA had the best diagnostic performance for viral hepatitis followed by TCA and GCDC A. Combination of these parameters had a better sensitivity and specificity for predicting chronic viral hepatitis with AUROC $=0.847$.

These results were in contrast of [7] where in their study they found that there was no significant difference between the two groups regarding all types of bile acids, and they explained this by the small number of NAFLD group included in their study which was 13 only.

\section{Conclusion}

In conclusion, the present study revealed that the compositions of serum BA including primary, secondary, and conjugated ones using LC-MS/MS could differentiate between chronic viral hepatitis and NAFLD patients, and they might be potential distinguishing biomarkers for this purpose. Further studies are warranted to study the efficacy of BAs as noninvasive biomarker for diagnosis of NAFLD.

\section{Abbreviations \\ BAs: Bile acids; CDCA: Chenodeoxycholic acid; CA: Cholic acid; TCA: Taurocholic acid; GCA: Glycocholic acid; TCDCA: Taurochenodeoxycholic acid; GDCA: Glycochenodeoxycholic acid; LCA: Lithocholic acid; DCA: Deoxycholic acid; UDCA: Ursodeoxycholic acid; HCV: Hepatitis C virus: HBV: Hepatitis B virus; LC: Liver cirrhosis; HCC: Hepatocellular carcinoma; NAFLD: Nonalcoholic fatty liver disease; NASH: Nonalcoholic steatohepatitis; UPLC-MS/MS: Ultra-performance liquid chromatography tandem mass spectrometer; IBA: Individual bile acids; GCA: Glycocholic acid; GCDCA: Glycochenodeoxycholic acid; GUDCA: Glycoursodeoxycholic acid; TDCA: Taurodeoxycholic acid; TUDCA: Tauroursodeoxycholic acid; TLCA: Taurolithocholic acid; HBs Ag: Hepatitis B surface antigen; AST: Aspartate aminotransferase; ALT: Alanine aminotransferase; T BIL: Bilirubin total; ALP: Alkaline phosphatase; GGT: Gamma-glutamyl transpeptidase; AFP: Alfafetoprotein; LB: Liver biopsy}

\section{Acknowledgements}

All authors are greatly indebted to members of Biochemistry and Molecular Diagnostics and Hepatology and gastroenterology departments of National Liver Institute, Menoufia University, Egypt. Also deep thanks to members of Zoology Department, Faculty of Science, Menoufia University, Egypt.

\section{Authors' contributions}

AE: initiated the project, designed and implemented the study for application, drafted and revised the paper; MO: analyzed the data, drafted and revised the paper; EA: analyzed the data, drafted and revised the paper; MB: analyzed the data, drafted and revised the paper; HE: analyzed the data, drafted and revised the paper. All authors have read and approved the manuscript.

\section{Funding}

This research and the publication were completely funded by all authors.

\section{Availability of data and materials}

Data used to support the findings of this study are included within the article.

\section{Ethics approval and consent to participate}

The study was conformed to the ethical guidelines of the 1975 Declaration of Helsinki and was approved by the institutional review board of National Liver Institute, Menoufia University, Egypt (NO. 00210/2020). Written informed consents were obtained from all participants.

\section{Consent for publication}

Written informed consents were obtained from both patients and control. Patients involved in this study agree for publication of data.

\section{Competing interests}

The authors declare that they have no competing interests.

\section{Author details}

'Department of Biochemistry and Molecular Diagnostics, National Liver Institute, Menoufia University, Shebeen El-Kom, Egypt. ${ }^{2}$ Department of Zoology, Faculty of Science, Menoufia University, Shebeen El-Kom, Egypt. ${ }^{3}$ Department of Hepatology and Gastroenterology, National Liver Institute, Menoufia University, Shebeen El-Kom 32511, Egypt.

Received: 28 July 2020 Accepted: 14 September 2020

Published online: 04 November 2020

References

1. Hanafi NI, Mohamed AS, Sheikh Abdul Kadir SH, Othman MH (2018) Overview of bile acids signaling and perspective on the signal of 
ursodeoxycholic acid, the most hydrophilic bile acid, in the heart. Biomolecules 8(4):159

2. Šarenac TM, Mikov M (2018) Bile acid synthesis: from nature to the chemical modification and synthesis and their applications as drugs and nutrients. Front Pharmacol 9:939

3. Murakami E, Wang T, Park Y, Hao J, Lepist El, Babusis D, Ray AS (2015) Implications of efficient hepatic delivery by tenofovir alafenamide (GS-7340) for hepatitis B virus therapy. Antimicrob Agents Chemother 59(6):3563-3569

4. Chow MD, Lee YH, Guo GL (2017) The role of bile acids in nonalcoholic fatty liver disease and nonalcoholic steatohepatitis. Mol Asp Med 56:34-44

5. Chiang JY (2017) Recent advances in understanding bile acid homeostasis. F1000Research 6:2029

6. Halilbasic E, Claudel T, Trauner M (2013) Bile acid transporters and regulatory nuclear receptors in the liver and beyond. J Hepatol 58:155-168

7. Sugita T, Amano K, Nakano M, Masubuchi N, Sugihara M, Matsuura T (2015) Analysis of the serum bile acid composition for differential diagnosis in patients with liver disease. Gastroenterol Res Pract 717431. https://doi.org/ 10.1155/2015/717431

8. Brunt EM, Janney CG, Bisceglie AM (1999) Non-alcoholic steatohepatitis: a proposal for grading and staging the histological lesions. Am J Gastroenterol 94:2467-2474

9. Abozeid M, Alsebaey A, Abdelsameea E, Othman W, Elhelbawy M, Rgab A et al (2018) High efficacy of generic and brand direct acting antivirals in treatment of chronic hepatitis C. Int J Infect Dis 75:109-114. https://doi.org/ 10.1016/j.jij. 2018.07.025

10. Alsebaey A, Badr R, Abdelsameea E, Amer MO, Eljaky MA, El-Azab G and SalamaM (2019): King's fibrosis, fibrosis index, GPR, and ALBI score are useful models for liver fibrosis in chronic hepatitis B patients pre- and posttreatment. Hepat Mon; 19(11):e96081.

11. Cotter TG, Mary Rinella M (2020) Nonalcoholic fatty liver disease 2020: the state of the disease. Gastroenterology 158(7):1851-1864. https://doi.org/10 1053/j.gastro.2020.01.052 Epub 2020 Feb 13

12. Ratziu V, Bellentani S, Cortez-Pinto H et al (2010) A position statement on NAFLD/NASH based on the EASL 2009 special conference. J Hepatol 53: 372-384

13. Luo L, Aubrecht J, Li D, Warner RL, Johnson KJ, Kenny J, Colangelo JL (2018) Assessment of serum bile acid profiles as biomarkers of liver injury and liver disease in humans. PLoS One 13(3):e0193824

14. Wang X, Xie G, Zheng X, Huang F, Wang Y, Yao C, Jia W, Liu P (2016) Serum bile acids are associated with pathological progression of hepatitis Binduced cirrhosis. J Proteome Res 15(4):1126-1134

15. Yin P, Wan D, Zhao C, Chen J, Zhao X, Wang W, Lu X, Yang S, Gu J, Xu G (2009) A metabonomic study of hepatitis B-induced liver cirrhosis and hepatocellular carcinoma by using RP-LC and HILIC coupled with mass spectrometry. Mol BioSyst 5(8):868-876

16. Makino I, Hashimoto H, Shinozaki K, Yoshino K, Nakagawa S (1975) Sulfated and nonsulfated bile acids in urine, serum, and bile of patients with hepatobiliary diseases. Gastroenterology 68(3):545-553

17. Wang C, Zhu C, Shao L, Ye J, Shen Y, Ren Y (2019) Role of bile acids in dysbiosis and treatment of nonalcoholic fatty liver disease mediators of inflammation. 2019(7659509):13

18. Minnullina ZS, Kiyashko SV, Ryzhkova OV, Sayfutdinov RG (2015) Bile acids serum levels in patients with nonalcoholic fatty liver disease. Kazan Med J 96(3):354-358

19. Kalhan SC, Guo L, Edmison J, Dasarathy S, McCullough AJ, Hanson RW, Milburn M (2011) Plasma metabolomic profile in non-alcoholic fatty liver disease. Metabolism. 60(3):404-413

\section{Publisher's Note}

Springer Nature remains neutral with regard to jurisdictional claims in published maps and institutional affiliations.

\section{Submit your manuscript to a SpringerOpen ${ }^{\circ}$ journal and benefit from:}

- Convenient online submission

- Rigorous peer review

- Open access: articles freely available online

- High visibility within the field

- Retaining the copyright to your article

Submit your next manuscript at $\boldsymbol{\nabla}$ springeropen.com 